Revista de Comunicación y Salud, 2020, Vol. 10, no 2, pp. 323-342

Editado por Cátedra de Comunicación y Salud

ISSN: 2173-1675

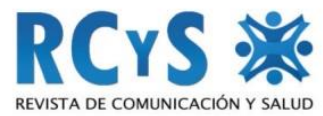

Enviado 15/08/2020

Aprobado 23/09/2020

\title{
RIEGOS DE LA COMUNICACIÓN DIGITAL EN LA TRANSICIÓN A LA DOCENCIA NO PRESENCIAL
}

\author{
Risks of digital communication in the transition to non-presential teaching \\ Jesús Cuevas Salvador ${ }^{1}$ \\ Universidad de Zaragoza. España \\ jesuscs@unizar.es
}

\section{Resumen}

El impacto del confinamiento por el Covid-19 ha transformado el modelo de educación acelerando nuevos canales de comunicación basados en la digitalización. De forma repentina el alumnado debía continuar su proceso de aprendizaje desde casa, la actividad docente basada en la presencia del profesor y el alumnado en el mismo espacio se ha transformado para convertirse en un espacio digital. El nuevo modelo no ha estado exento de complicaciones, esta investigación ha permitido analizar cómo se ha desarrollado el proceso de enseñanza aprendizaje a través de la comunicación digital para descubrir los nuevos riesgos que se deben prevenir en la educación online. Aplicando un cuestionario online el profesorado ha expresado su experiencia como docente virtual y ha definido riesgos que se deben afrontar y superar: la brecha digital, la carencia de competencias digitales, el docente como transmisor, la desmotivación y el abandono del alumnado. Se ha verificado la hipótesis que establece relación entre el grado de comunicación digital y el grado de desempeño de la docencia en la modalidad no presencial. El escenario educativo es diseñado por los nuevos canales de comunicación digital, basados en la necesidad de la alfabetización digital para el alumnado y para el profesorado, e incorporar en la didáctica la monitorización de las nuevas aplicaciones digitales.

Palabras clave: comunicación digital, didáctica, brecha digital, enseñanza, docencia no presencial, competencias digitales, aprendizaje.

\begin{abstract}
The impact of confinement by Covid-19 has transformed the education model by accelerating new communication channels based on digitization. Suddenly the students had to continue their learning process from home, the teaching activity based on the
\end{abstract}

\footnotetext{
1 Jesús Cuevas Salvador. Universidad de Zaragoza. jesuscs@unizar.es Licenciado en Ciencias Políticas y Sociología, Doctorado por la Universidad de Zaragoza, en la actualidad profesor en el Máster en Profesorado de la Facultad de Educación.
} 
presence of the teacher and the students in the same space has been transformed to become a digital space. The new model has not been without complications. This research has allowed us to analyze how the teaching-learning process has developed through digital communication to discover the new risks that must be prevented in online education. By applying an online questionnaire, the teachers have expressed their experience as a virtual teacher and have defined risks that must be faced and overcome: the digital divide, the lack of digital skills, the teacher as transmitter, demotivation and the abandonment of students. The hypothesis that establishes a relationship between the degree of digital communication and the degree of performance of teaching in the non-face mode has been verified. The educational scenario is designed by the new digital communication channels, based on the need for digital literacy for students and teachers, and incorporating the monitoring of new digital applications into teaching.

Keywords: digital communication, didactic, digital divide, teaching, non-face-to-face teaching, digital skills, learning.

\section{Cómo citar el artículo}

Cuevas Salvador, J. (2020). Riegos de la comunicación digital en la transición a la docencia no presencial. Revista de Comunicación y Salud, 10 (2), 323-342. doi: https://doi.org/10.35669/rcys.2020.10(2).323-342

\section{INTRODUCCIÓN}

\subsection{Contexto normativo de la transición educativa, desde la presencialidad en las aulas hacia la modalidad no presencial}

La Organización Mundial de la Salud (OMS) elevó el 11 de marzo de 2020 la emergencia de salud pública ocasionada por la enfermedad por coronavirus en el 2019 (COVID-19) a pandemia internacional. La rapidez de la evolución de los hechos ha requerido de medidas inmediatas para proteger la salud y seguridad de los ciudadanos, para contener la progresión de la enfermedad, reforzar el sistema de salud pública para cubrir las necesidades de los ciudadanos afectados y evitar riesgos en sus derechos.

Para hacer frente a la crisis sanitaria, y en el contexto de la Ley Orgánica 4/1981, de 1 de junio, de los estados de alarma, el 14 de marzo de 2020 por medio del Real Decreto 463/2020 se declara el estado de alarma en España, para la gestión de la crisis sanitaria ocasionada por el COVID-19.

Las medidas no suponen la suspensión de ningún derecho fundamental, tal como prevé el artículo 55 de la Constitución española. Durante la vigencia del estado de alarma las personas deberán limitar el circular por las vías de uso público según indica el artículo 7, y en el artículo 9 del Real Decreto 463/2020 del 14 de marzo, se describen las medidas de contención en el ámbito educativo y de la formación: 
Riegos de la comunicación digital en la transición a la docencia no presencial

- Se suspende la actividad educativa presencial en todos los centros y etapas, ciclos, grados, cursos y niveles de enseñanza contemplados en el artículo 3 de la Ley Orgánica 2/2006, de 3 de mayo, de Educación, incluida la enseñanza universitaria, así como cualesquiera otras actividades educativas o de formación impartidas en otros centros públicos o privados.

- Durante el período de suspensión se mantendrán las actividades educativas a través de las modalidades a distancia y online, siempre que resulte posible.

Dentro de la normativa estatal del Ministerio de Educación y Formación Profesional, la Orden EFP/365/2020, de 22 de abril, por la que se establecen el marco y las directrices de actuación para el tercer trimestre del curso 2019-2020 y el inicio del curso 2020-2021, ante la situación de crisis ocasionada por el COVID-19. ha adoptado medidas que definen y amplían progresivamente las actuaciones en el periodo de confinamiento en la Formación Profesional (FP):

- Cuidado y bienestar del alumnado y las familias en relación con la prevención de higiene y promoción de la salud, ayudas económicas y apoyo emocional.

- Calendario escolar y actividades lectivas incluyendo la suspensión de la actividad educativa presencial en todos los centros y etapas y sustitución por las modalidades a distancia y online.

- Recursos educativos para la enseñanza-aprendizaje. Programas digitales para impulsar la transformación tecnológica de la educación, recursos para el aprendizaje en línea, portal web para facilitar el acceso a recursos, herramientas y aplicaciones al profesorado, las familias y el alumnado, información para el aprendizaje a distancia

- Las pruebas de evaluación adquieren el carácter de diagnóstico y formativo, las pruebas presenciales deben ser sustituidas por pruebas con recursos telemáticos.

La Orden EFP/361/2020, de 21 de abril, por la que se adoptan medidas excepcionales en materia de flexibilización de las enseñanzas de Formación Profesional del Sistema Educativo y de las enseñanzas de Régimen Especial, tiene por objeto el establecimiento de medidas excepcionales durante el curso escolar 2019-2020 en materia de ordenación y organización de las enseñanzas de formación profesional del sistema educativo, así como enseñanzas artísticas y deportivas:

- La brecha digital del alumnado. Para no aumentar la desigualdad en el acceso al aprendizaje hay que garantizar el acceso al aprendizaje e igualdad de posibilidades desde casa: ordenador, auriculares, micros, programas y conexión a internet de alta velocidad.

- Proyectos cooperativos. Creación de proyectos motivadores para poder interactuar y cooperar con los estudiantes telemáticamente y ello conlleva modificar el sistema de evaluación.

- Prácticas de taller online. Reinvención de las prácticas de taller y de laboratorio para adaptarlas a modalidad online. 
Riegos de la comunicación digital en la transición a la docencia no presencial

- Prácticas en un centro de trabajo. Medidas relativas a las prácticas de formación en el centro de Trabajo o de FP Dual, por estar afectadas por Expediente de Regulación de Empleo o el Expediente de Regulación Temporal de Empleo.

- Recursos tecnológicos. ¿Tienen los docentes los recursos técnicos necesarios? Recursos humanos y tecnológicos para afrontar la digitalización, el seguimiento individual del alumnado, la formación digital.

También la Universidad de Zaragoza (2020) ha transformado la docencia presencial en docencia no presencial en resoluciones de 13 y 15 de marzo de 2020, quedando suspendidas las clases presenciales de conformidad con el Protocolo de actuación ante la alerta sanitaria por el COVID-19. La alteración de la docencia afecta principalmente a las programaciones, las metodologías docentes y a las pruebas de evaluación.

\subsection{La digitalización, los nuevos canales de comunicación en la educación}

La suspensión de la actividad educativa presencial ha provocado de forma imprevista una alteración del desarrollo del curso escolar 2019-2020, obligando a realizar un gran esfuerzo por el conjunto de la comunidad educativa para poder dar continuidad a la actividad lectiva a través de otras modalidades de enseñanza y aprendizaje, sin clases presenciales.

De un día para otro se ha pasado de contemplar la teleformación como una modalidad de enseñanza opcional a ser considerada la opción principal, una modalidad obligatoria en todo sistema educativo y que ha supuesto el cambio de mentalidad en el profesorado y alumnado y adaptar un proceso de enseñanza aprendizaje obligatoriamente presencial a un nuevo sistema a distancia y online. El nuevo escenario educativo el protagonismo es adquirido por las Tecnologías de la Comunicación y de la Información (TIC) y la comunicación digital para diseñar el nuevo modelo basado en la digitalización de la educación.

¿Cómo era el uso de las TIC en las aulas antes del Covid-19? ¿Estaba preparado el sistema educativo para dar el salto a la transformación digital en una semana? En relación con el uso de las TIC, BlinkLearning (2020) presentó los resultados de la investigación "V Estudio sobre el uso de las TIC en la Educación" realizada en Latinoamérica y España en los meses previos al inicio de COVID-19, mostrando una radiografía sobre el nivel de digitalización del sistema educativos. El objetivo del estudio se ha basado en averiguar los dispositivos más utilizados, los principales retos y dificultades encontradas en el uso de las TIC, las ventajas en el proceso de enseñanza aprendizaje, actitud y motivación ante las TIC y el nivel de formación digital. Las principales conclusiones que se extraen de este estudio destacan las ventajas de introducir los recursos tecnológicos en la educación:

- El uso de las TIC significa el acceso a un mayor número de contendidos y recursos.

- El $40 \%$ de los docentes encuestados opina que las TIC fomentan el aprendizaje autónomo.

Revista de Comunicación y Salud, 2020, Vol. 10, nº 2, pp. 323-342 
Riegos de la comunicación digital en la transición a la docencia no presencial

- Antes de la pandemia, la formación del profesorado en TIC era deficitaria,

- Las TIC motivan al alumnado, sobretodo el uso del móvil para comunicarse y acceder a la información.

- Los mayores retos para conseguir la digitalización educativa son la formación del profesorado, el problema de la conectividad y el acceso a dispositivos tecnológicos.

- La motivación sigue siendo el motor del alumnado y del profesorado para la mejora de la educación.

- En el alumnado el principal déficit es la dificultad para seleccionar fuentes de información fiable y falta de alfabetización digital para utilizar aplicaciones

\subsection{Definición del problema y propósito de la investigación}

La utilización de las TIC en la educación según Gómez, Contreras y Gutiérrez (2020) ha propiciado la discusión sobre las ventajas y desventajas de su incorporación: entre las mayores ventajas, el uso de las TIC facilitan el proceso de enseñanza-aprendizaje por el acceso a una mayor cantidad mayor de información, también facilitan el contacto con muchas personas, como desventaja señalan la accesibilidad desigual del alumnado al uso de las TIC.

El estudio de Torrecillas, Vázquez, Suárez, y Fernández (2020) indica que la inmersión digital de la sociedad española es una realidad, el uso de las pantallas de los smartphone está cada vez más presente tanto en la niñez como en la adolescencia, consiguiendo sustituir a la televisión en su influencia socializadora, integrándose en la forma de entretenerse, relacionarse y de aprender. Según la Fundación Telefónica (2016), el 81,9\% de los hogares españoles está conectado a internet y el $95 \%$ de las personas de 10 a 15 años navega por internet.

El Covid-19 está cuestionando la forma en que se imparte la educación, la forma en que el alumnado aprende. La Organización de las Naciones Unidas para la Educación, la Ciencia y la Cultura (Unesco, 2013) describe que establecer conexiones entre educación y TIC es más que hablar de ordenadores, aplicaciones, herramientas y programas, significa la oportunidad de reflexionar sobre cómo se piensa en educación y de qué manera el alumnado y docentes aprenden y enseñan.

La irrupción del Covid-19 ha acelerado el debate sobre los impactos del uso de las TIC en los procesos de enseñanza-aprendizaje, para Sierra (2020) el escenario es de incertidumbre y afecta a todas las dimensiones humanas, los comportamientos cotidianos, los modelos de la realidad basados en la necesidad de la conectividad y el riesgo de la brecha digital y el desarrollo del potencial de cada persona en la responsabilidad de sus elecciones

Ante el nuevo escenario de incertidumbre Cabrera (2020) opina sobre la crisis del Covid-19 como motor de generación de cambio social fortaleciendo el sistema educativo al hacer evaluación de las debilidades y oportunidades. Esta investigación 
hace una radiografía de cómo ha sido el proceso de la comunicación entre los docentes y el alumnado durante la cuarentena, descubriendo los riesgos cuando los espacios físicos de socialización y aprendizaje de la escuela se trasladan al hogar de cada estudiante, cuando la escuela y la casa se convierten en el mismo lugar.

En el contexto del estado de la cuestión presentado, surgen preguntas que muestran la relevancia de este estudio ¿Qué riesgos aparecen en el proceso de enseñanzaaprendizaje, desde casa, para el profesorado y para el alumnado? ¿Las aplicaciones digitales se han convertido en la vía para la comunicación didáctica? ¿La comunicación digital es compatible con el desarrollo del currículo?

La comunicación didáctica consiste en la comunicación y transmisión de información entre docente y el estudiante en el contexto del currículo, según López (2020) el currículo organiza el enfoque del aprendizaje por competencias para conseguir afrontar las siguientes cuestiones:

- Impartir contendidos del Sistema Nacional de Cualificaciones. Los contenidos teóricos pasarán más a los prácticos, de forma que los alumnos focalizaran en trabajos y ensayos, suprimiendo exámenes.

- Motivar, hacer frente a la dificultad de tener que motivar un grupo clase online, para generar vínculos y responsabilidades.

- Metodologías y actividades para activar el aprender a aprender, aprendizaje significativo y por competencias.

- ¿Es fiable el aprendizaje del alumnado? Una de las principales dudas es comprobar que el alumnado ha conseguido asimilar el temario de las asignaturas y verificar que el aprendizaje ha sido igual al de las clases presenciales.

- ¿Es igual de fiable un examen telemático que uno presencial? Consigue medir el conocimiento del alumnado y se asegura de que el aluno no comete fraude.

\section{OBJETIVOS}

Los objetivos de la investigación están relacionados con las funciones de la labor docente definidas en la Ley Orgánica para la Mejora de la Calidad Educativa y relevantes para impartir enseñanzas de formación profesional:

- El proceso de enseñanza de los módulos que componen la programación.

- La evaluación del proceso de aprendizaje del alumnado, así como la evaluación de los procesos de enseñanza.

- La tutoría de los alumnos, la dirección y orientación de su aprendizaje y el apoyo en su proceso educativo.

- La orientación educativa, académica y profesional de los alumnos, en colaboración con los departamentos especializados.

- La atención al desarrollo intelectual, afectivo, social y moral del alumnado.

- La contribución al clima en el centro y el aula de respeto, tolerancia, participación, y libertad para el fomento de los valores de una ciudadanía democrática.

Revista de Comunicación y Salud, 2020, Vol. 10, nº 2, pp. 323-342 
Riegos de la comunicación digital en la transición a la docencia no presencial

- La promoción, organización y participación en actividades complementarias, dentro o fuera del recinto educativo.

- La participación en los planes de evaluación que determinen las Administraciones Educativas o los propios centros.

La formación inicial pedagógica y didáctica para el profesorado es obligatoria por medio de Postgrado y la incorporación en los centros se desarrolla bajo la tutoría de profesores experimentados. En la formación permanente del profesorado las Administraciones educativas promoverán la actualización y utilización de las tecnologías de la información y la comunicación y a formación de lenguas extranjeras de todo el profesorado, también fomentar programas de investigación e innovación que acompaña a la función docente.

También las Administraciones educativas prestarán una atención prioritaria a la observación y mejora de las condiciones en que el profesorado realiza su trabajo y al estímulo de una creciente consideración y reconocimiento social de la función docente. El objetivo general de la investigación, en el contexto de suspensión de la actividad educativa presencial, manteniendo las actividades educativas a través de las modalidades a distancia y online: analizar el proceso de adaptación de la modalidad de docencia presencial a la modalidad de docencia no presencial de la actividad docente en los centros educativos de Formación Profesional durante el confinamiento por el estado de emergencia sanitaria producida por el COVID-19.

¿Qué esfuerzos ha realizado el profesorado para mantener activa la docencia a distancia y online? ¿Cuáles son los riesgos de la docencia a distancia y online? ¿Cómo afecta al diseño de metodologías y actividades en el aprendizaje del alumnado? Ante estas cuestiones, la investigación seguirá los siguientes objetivos específicos:

- Conocer los roles del profesorado ante la docencia no presencial.

- Averiguar el impacto de la docencia no presencial en el alumnado.

- Analizar los riesgos del nuevo escenario educativo.

La investigación permitirá conocer las opiniones del profesorado ante los riesgos de la nueva modalidad de docencia en comparación con el modelo de enseñanza presencial, el uso de las nuevas tecnologías de la información y la digitalización de la educación, averiguar la opinión sobre la fiabilidad del aprendizaje del alumno, el grado de alfabetización digital en el alumnado y en el profesorado, las claves para el éxito de las metodologías y actividades, los canales de comunicación entre profesor y el alumnado y la comunicación entre el alumnado, el proceso para captar la atención del alumnado y la regulación de su aprendizaje. 
Riegos de la comunicación digital en la transición a la docencia no presencial

\section{METODOLOGÍA}

\subsection{Población y diseño de la muestra seleccionada}

En la población objeto de estudio participa el profesorado de Formación Profesional de 15 Institutos de Educación Secundaria repartidos en las provincias de Zaragoza, Huesca y Teruel de la Comunidad Autónoma de Aragón. Entre los 26 tipos de familias de Formación Profesional específicas, el profesorado participante pertenece a las familias profesionales de Administración y Gestión, Comercio y Marketing, Hostelería y Turismo, Servicios Socioculturales y a la Comunidad, también se incluye al profesorado del módulo transversal de Formación y Orientación Laboral.

En la estrategia del diseño de la muestra, con el objetivo de minimizar el error muestral, permitiendo el rigor y la validez científica, el requisito imprescindible que define la muestra es la selección del profesorado que debe realizar la tarea de tutorizar al alumnado que está cursando la asignatura Practicum II dentro del programa del Máster habilitante para ejercer la profesión docente, Máster en Educación Secundaria Obligatoria, Bachillerato, Formación Profesional y Enseñanzas de Idiomas, Artísticas y Deportivas, impartido en la Facultad de Educación de la Universidad de Zaragoza, durante el curso 2019/2020. El Máster se compone de 17 especialidades, la unidad de análisis o muestra seleccionada, donde se realizará la recolección de datos, está formada por los tutores del alumnado matriculado en la especialidad de Formación Profesional de las familias de Administración, Marketing, Turismo, Servicios a la Comunidad y Formación y Orientación laboral (FOL).

El tamaño de la muestra tiene carácter intencional, se trata de un diseño muestral no probabilístico y opinático (Wood y Smith, 2018), se compone por 27 profesores, en la distribución por género, el $85 \%$ es femenino y el $15 \%$ es de género masculino, profesorado que ejerce la labor de tutorización del Practicum II en centros de Formación Profesional en la enseñanza de ciclos formativos de las familias profesionales de administración y gestión, Comercio y marketing, Hostelería y Turismo, Servicios socioculturales y a la comunidad. Para Barroso y Cabero (2013), la unidad muestral formada por el profesorado está capacitado para analizar el papel que desempeñan las nueva tecnologías tanto en el profesorado como en el alumnado, para averiguar las aplicaciones digitales utilizadas y estudiando las competencias digitales más idóneas para el desarrollo curricular.

Las medidas y obligaciones derivadas del Real Decreto 463/2020, de 14 de marzo, y las sucesivas prórrogas del estado de alarma que ha sido preciso aprobar, con la debida autorización del Congreso de los Diputados, impidiendo no solo la actividad educativa presencial, sino también los periodos de formación en centros de trabajo o de prácticas de diversas enseñanzas universitarias y no universitarias, actividades de prácticas necesarias para concluir los estudios y obtener las correspondientes titulaciones. Con estas indicaciones el profesorado de Formación Profesional tutorizó al alumnado del Practicum II de forma no presencial, en confinamiento estricto, durante el periodo del 15 de abril al 22 de mayo de 2020.

Revista de Comunicación y Salud, 2020, Vol. 10, nº 2, pp. 323-342 
Lo esencial de la asignatura Practicum II es aproximarse al entorno docente de un Centro de Formación Profesional. La asistencia no presencial al centro de prácticas durante la totalidad del período establecido es condición necesaria para que el alumnado pueda ser evaluado en la asignatura del Máster. Los resultados de aprendizaje al finalizar la asignatura (Universidad de Zaragoza, 2020):

- Características de los alumnos y de la clase, entendida como grupo formativo y social, las estrategias didácticas utilizadas por el profesor y los principales problemas de enseñanza-aprendizaje detectados.

- Diseñar, poner en práctica y evaluar secuencias didácticas que estén fundamentadas en las teorías de enseñanza-aprendizaje, incorporando elementos de innovación didáctica, y propuesta de mejoras basadas en el análisis de la experiencia.

- Identificar los contenidos que se trabajan en el aula virtual durante el periodo de prácticas, relacionándolos con las programaciones de ciclo y con los documentos curriculares oficiales.

- Identificar las dificultades de aprendizaje del alumnado, analizando sus causas y estableciendo implicaciones sobre el proceso de enseñanza.

El Practicum II capacita al alumnado, junto con el resto de asignaturas, para ejercer la función docente en el ámbito de la formación profesional con garantías de poder plantear diferentes propuestas para evaluar, innovar e investigar sobre los propios procesos de enseñanza en el objetivo de la mejora continua en el desempeño docente y de la tarea educativa del centro.

La Formación Profesional, en el sistema educativo, prepara al alumnado para ejercer la actividad profesional y facilitar su adaptación a lo largo de la vida al mercado laboral cambiante, contribuir a su desarrollo personal y al ejercicio de una ciudadanía democrática y permitir un itinerario en el aprendizaje a lo largo de la vida a través del sistema de formación profesional para el empleo y su progresión en el sistema educativo. Dentro de los principios generales de la Ley Orgánica 8/2013, de 9 de diciembre, para la mejora de la calidad educativa (LOMCE), que modifica la Ley Orgánica 2/2006, de 3 de mayo, e Educación (LOE):

La formación profesional comprende el conjunto de acciones formativas que capacitan para el desempeño cualificado de las diversas profesiones, el acceso al empleo y la participación activa en la vida social, cultural y económica. Incluye las enseñanzas propias de la formación profesional inicial, las acciones de inserción y reinserción laboral de los trabajadores así como las orientadas a la formación continua en las empresas, que permitan la adquisición y actualización permanente de las competencias profesionales (p.37)

En relación con el tema de esta investigación y la Formación Profesional, destacar tres ámbitos que destaca la LOMCE, como ejes para la transformación del sistema educativo: las tocologías de la información y de la comunicación, el fomento del plurilingüismo y la modernización de la Formación Profesional española. 
Riegos de la comunicación digital en la transición a la docencia no presencial

La estructura de las familias profesionales se compone de un conjunto de cualificaciones formadas según la Ley 5/2002 de 19 de junio, de las Cualificaciones y de la Formación Profesional, las cualificaciones son el conjunto de competencias profesionales con significación para el empleo que pueden ser adquiridas mediante formación modular y a través de la experiencia laboral. Los módulos profesionales organizan la formación para adquirir una cualificación profesional, organizan el proceso de aprendizaje y proporcionan un conjunto de conocimientos, habilidades y aptitudes que completan una formación y capacitación para el desempeño de una profesión. La evaluación del aprendizaje del alumnado en los ciclos de Formación Profesional Básica y en los ciclos formativos de Grado Medio y Superior se realizará por módulos profesionales y, en su caso, por materias o bloques, de acuerdo con las condiciones que el Gobierno determine reglamentariamente.

\subsection{Hipótesis y variables}

¿El confinamiento significaba el fin del curso académico, de la docencia y del proceso de enseñanza aprendizaje? La suspensión de la actividad educativa presencial en todos los centros educativos debía ser sustituida por la modalidad a distancia u online. Ante la nueva fórmula de enseñanza y la falta de un plan de contingencia debido a la inmediatez de la pandemia ¿Cuál ha sido la respuesta del alumnado y de los docentes?

¿Ante la irrupción del Covid-19, los centros educativos, el alumnado y los docentes estaban preparados para afrontar con éxito el proceso de enseñanza aprendizaje virtual? El Covid-19 ha llegado cuando todos los centros educativos están informatizados, cuando la mayoría de los hogares disponen de ordenador y conexión a internet y cuando casi la totalidad de las personas disponen de teléfono móvil. El Covid19 ha llegado en el contexto de democratización del uso de las TIC para el desarrollo de la sociedad de la información y de la comunicación, y la digitalización de la sociedad para la mejora de la competitividad.

La investigación se ha realizado durante el periodo lectivo de a asignatura Practicum II adaptada a la no presencialidad, para hacer un análisis de la realidad sobre cómo se ha desarrollado el proceso de enseñanza aprendizaje, averiguando cuáles han sido los canales de comunicación entre el profesorado y el alumnado en la modalidad no presencial y cuáles son los riesgos más inminentes a los que hay que hacer frente. Para averiguar estas cuestiones se ha definido un tipo de hipótesis causal y relacional: El grado de comunicación digital influye en el grado desempeño de la docencia en la modalidad no presencial.

La preocupación en la investigación se centra en las posibilidades del liderazgo del docente para la gestión del aula virtual, y si los nuevos roles del liderazgo docente están directamente relacionados con la mentalidad para la comunicación digital (Lankshear y Knobel,2009), así a mayor comunicación digital mayor eficiencia en la docencia. El impacto de la docencia digital o virtual en el proceso de enseñanza

Revista de Comunicación y Salud, 2020, Vol. 10, oㅡ 2, pp. 323-342 
Riegos de la comunicación digital en la transición a la docencia no presencial

aprendizaje se analizará parcialmente, siendo una cuestión relevante para futuras investigaciones.

La comunicación digital (Pérez, 2017; Romero y Rivera, 2019) significa una nueva forma de conectividad, de comprensión y creación de información. Interviene el aprendizaje y habilidades para el uso de las TIC o tecnología digital, incluye la habilidad para localizar, almacenar, recuperar, presentar, investigar, analizar y evaluar la información, también elaborar contenidos e intercambiar información para comunicar y participar en redes de participación. El déficit en comunicación digital se convierte en analfabetización digital, por falta de acceso a las TIC y conexión a internet, generando exclusión social y brecha digital.

La variable independiente, "El grado de comunicación digital" es la clave para garantizar el éxito de la variable dependiente "Influye en el grado de desempeño de la docencia en la modalidad no presencial". Los indicadores que componen ambas variables, la variable independiente o competencia digital y variable dependiente 0 resultados de docencia, son establecidos por el Marco Europeo para la Competencia Digital de los Educadores (DigComEdu) describiendo el significado de los roles del docente digitalmente competente (European Commission, 2006).

\subsection{Métodos y técnicas para la recolección de datos}

Para averiguar cuáles son los riesgos de la comunicación digital en la docencia con modalidad no presencial, durante el confinamiento, se ha seleccionado la metodología descriptiva, con el objetivo de investigar los hechos por medio de una observación objetiva.

Los tutores del Practicum II no presencial ha permitido conocer la experiencia de establecer nuevos canales de comunicación entre el profesorado y el alumnado de los centros educativos. La información sobre cómo, cuándo, dónde, por qué y para qué de los canales utilizados se ha obtenido por medio de la técnica de observación y medición del cuestionario, diseñado con preguntas abiertas. Para Albert (2006), el cuestionario es una técnica estructurada que permite recoger abundante información por medio de la formulación de preguntas respecto a las variables a medir. El diseño del cuestionario, por medio de preguntas escritas, han permitido obtener los datos cualitativos y cuantitativos para el análisis del grado de alfabetización digital y su impacto en la modalidad no presencial de la docencia, una nueva realidad social que implica una nueva mentalidad.

En el diseño del cuestionario se ha seguido el razonamiento deductivo. La forma de respuesta individual del cuestionario se implementó al llegar al final del periodo del Practicum II, Según Berganza y Ruiz (2005) para conseguir que todo el alumnado se encuentre en la misma situación psicológica y entender lo mismo cuando se hacen lectura de las cuestiones. La Tabla 1, muestra las preguntas y sus correspondientes indicadores para analizar la hipótesis de la investigación. El cuestionario ha sido

Revista de Comunicación y Salud, 2020, Vol. 10, nº 2, pp. 323-342 
organizado, secuenciado y estructurado con el fin de que las respuestas puedan ser procesadas para obtener la información.

Tabla 1. Preguntas e indicadores del cuestionario

\begin{tabular}{l|l|}
\hline \multicolumn{1}{|c|}{ Indicadores } & \multicolumn{1}{c}{ Preguntas } \\
\hline - Autorregulación. & ¿Qué aplicaciones digitales se han utilizado en la \\
- Desmotivación y abandono. & comunicación digital y conductas inducidas? \\
\hline - Comunicación. & ¿Dónde reside el éxito de un profesor de FP en el \\
- Transmisor. & nuevo contexto de no presencialidad? \\
\hline - Aprendizaje a distancia. & ¿Hacia dónde va la educación? \\
\hline - Brecha digital. &
\end{tabular}

Fuente: elaboración propia

La metodología descriptiva y las técnicas de observación y medición por medio del cuestionario han permitido explicar la experiencia vivida por los docentes y el alumnado ante la modalidad de clases no presenciales, de forma inmediata e imprevista por causas de la pandemia del coronavirus. La sistematización por medio de la categorización y tabulación de la información obtenida a través de los cuestionarios ha garantizado la calidad científica de la investigación.

\section{DISCUSIÓN}

\subsection{Comunicación digital en los Institutos de Educación Secundaria}

De un día para otro la totalidad del alumnado del sistema educativo tuvieron que continuar su proceso de enseñanza aprendizaje en sus hogares, el espacio físico del aula se trasladó a las casas. El confinamiento ha transformado de forma imprevista la gestión de la educación y ha pasado de contar con bajos índices de digitalización a convertirse en el modelo futuro de educación. La cuarentena, desde los hogares, ha supuesto un entrenamiento forzado para hacer uso de las aplicaciones digitales.

La comunicación tradicional en las aulas ha pasado a ser una comunicación digital donde intervienen nuevas variables relacionadas con las TIC. La transición hacia el modelo educativo del futuro reside en la transformación de un modelo de aula presencial hacia un modelo de aula virtual. En este nuevo escenario la comunicación digital se convierte en la cuestión clave, los roles de los docentes se afianzan en ser los gestores del aprendizaje, facilitando las metodologías y actividades por medio de nuevos canales y observando que el alumnado alcanza a comprenderlos.

A través del cuestionario, el profesorado del Practicum II numeran cuáles son las herramientas que permiten establecer una comunicación digital con el alumnado de los IES de Formación Profesional. Los canales de comunicación más utilizados se visualizan en el Gráfico 1: 
Riegos de la comunicación digital en la transición a la docencia no presencial

- Correo electrónico. El 56\% utiliza el correo electrónico como vía de comunicación principal.

- Moodle. El 22\% ha nombrado la plataforma Moodle para la comunicación digital y para la gestión del aprendizaje. Moodle es útil para crear comunidades de aprendizaje en línea, se usa para la educación a distancia, compatible con el elearning y blended learning. Fomenta el constructivismo del conocimiento del alumnado.

- Clasroom-Meet. El 12\% ha indicado las aplicaciones de Google Classroom-Meet.

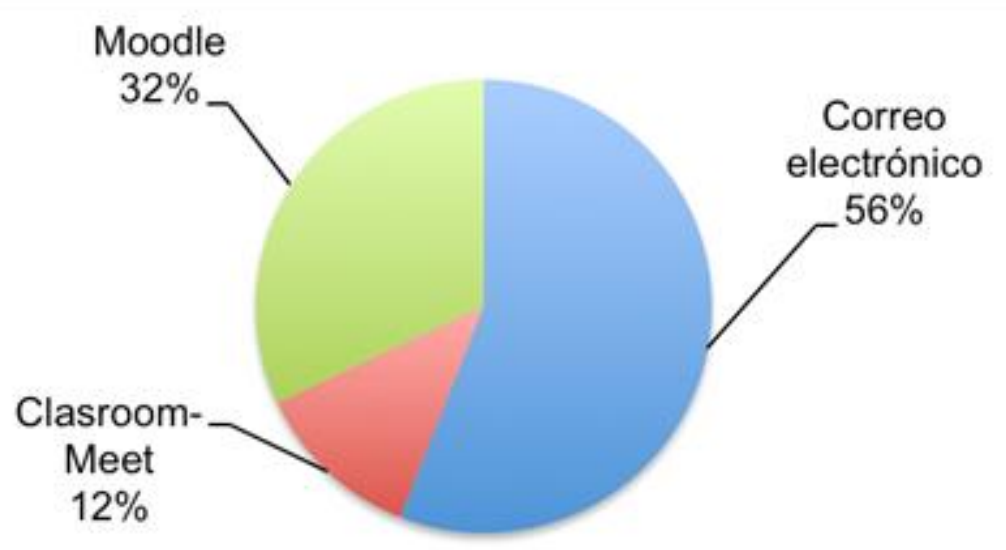

Grafico 1: Comunicación digital con el alumnado

Fuente: Elaboración propia

\subsection{Riesgos del liderazgo docente y actitud del alumnado}

Para que el docente sea tutor, facilitador, orientador, dinamizador, organizador, alejándose del rol tradicional que lo ubicaba como único transmisor del conocimiento, debe iniciar una escucha activa con el objetivo de detectar el conocimiento previo necesario en el alumnado para comprender las ideas, el nivel de complejidad cognitiva que deben disponer, la capacidad de transferencia y de desarrollo del pensamiento crítico y el tiempo efectivo que puedan destinar al esfuerzo del aprendizaje.

Los datos extraídos del cuestionario tras ser codificados y tabulados arrojan información relacionada con el nuevo liderazgo docente y la actitud del alumno ante el telestudio. El Gráfico 2 muestra la percepción del profesorado:

- Autorregulación. El $60 \%$ del profesorado percibe que el alumnado no ha abandonado el vínculo con el proceso de enseñanza-aprendizaje y con el aula virtual, ha demostrado competencias de para la adaptación y gestión del cambio basado en la autonomía e iniciativa personal, el sentido de la responsabilidad, la tenacidad y el espíritu de superación, la organización del tiempo, la planificación de las tareas y la toma de decisiones. Aunque es imposible generalizar la conducta por la variedad del alumnado FP Básica, Grado Medio, Grado Superior, 
Riegos de la comunicación digital en la transición a la docencia no presencial

se destaca la tendencia hacia la autonomía y la autorregulación del ritmo de aprendizaje.

- Desmotivación y abandono. La amenaza del abandono persiste en la Formación Profesional, el $40 \%$ del profesorado resalta que la desmotivación y las posibilidades de abandono permanece en el alumnado. Esta tendencia se acentúa principalmente entre el alumnado de FP Básica y Grado Medio y tiende a desaparecer en el alumnado de Grado Superior.

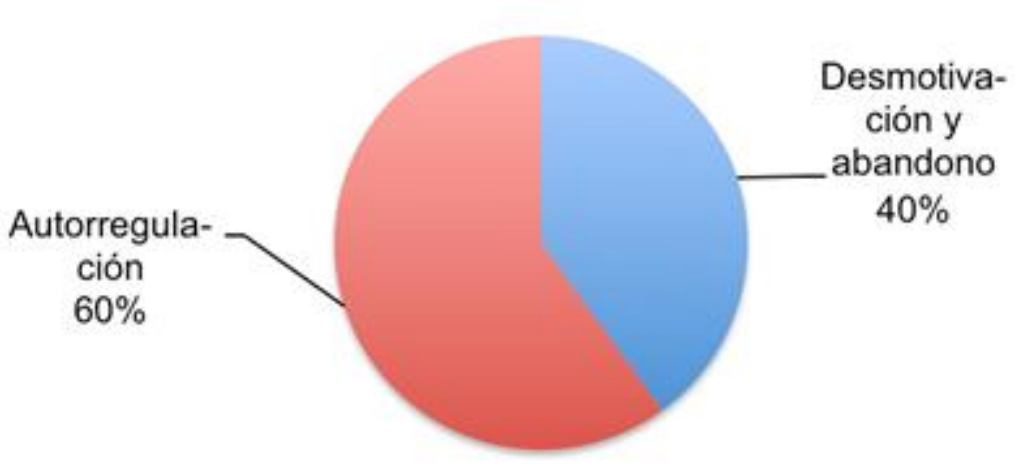

Grafico 2: Liderazgo docente y actitud alumnado

Fuente: Elaboración propia

\subsection{Riesgos del nuevo rol docente}

Hasta el confinamiento la tecnologización de la enseñanza consistió en un cambio de medios y no de métodos, debido a la inercia del diseño curricular y las programaciones didácticas de la educación presencial. Las metodologías se han basado principalmente en la clase magistral, el uso de la lengua escrita y el aprendizaje memorístico. Sin embargo la mayoría del alumnado es nativo digital, el uso de las nuevas tecnologías forma parte de sus vidas cotidianas.

El tele-estudio desde el hogar para los nativos digitales debe introducir recursos y metodologías alternativas al estudio de libros de texto y de la memorización y repetición. En el Gráfico 3, se presentan datos sobre el nuevo rol docente resaltando dos indicadores, comunicación y transmisión, presentándose como opuestos, también pueden ser complementarios:

- Comunicación. El $84 \%$ del profesorado ha sido contundente en relacionar digitalización de la educación con establecer nuevas modalidades de comunicación con el alumnado, comunicación para crear aulas virtuales, para activar la motivación, para gestionar la temporalización de metodologías y actividades basadas en competencias, para crear ambientes de estudio colectivos en la virtualidad.

- Transmisor. La transmisión de la información como vía para la generación de conocimiento y el docente erudito en su materia sigue siendo relevante para el $16 \%$ del profesorado. La cuestión es cómo adoptar este modelo a la didáctica que requiere la educación online, estableciendo los procedimientos más 
acertados para garantizar el conocimiento: la metodología necesaria para el aprendizaje por competencias.

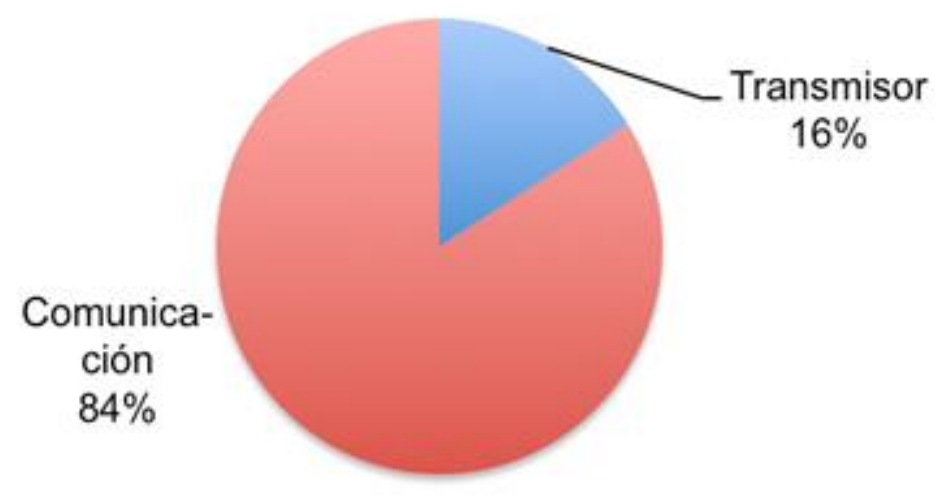

Grafico 3: Nuevo rol del docente

Fuente: Elaboración propia

El profesorado experto en sus materias, en la didáctica de las clases presenciales, de un día para otro se han visto en la necesidad de replantear la estructura curricular para adaptarla a la didáctica online, asumiendo el riesgo ante la incertidumbre del impacto incierto en el alumnado y dejando al descubierto la necesidad de iniciar una formación en competencias digitales aplicadas a la educación.

\subsection{Riesgos de la conectividad y brecha digital}

Un proceso de enseñanza-aprendizaje, con independencia de si la modalidad es presencial o no presencial, consiste en un intercambio de información utilizando canales de comunicación para permitir el feedback entre personas, el riesgo en este planteamiento es la brecha digital.

La brecha digital se relaciona con el acceso desigual al uso de las TIC de los grupos sociales. El uso de los teléfonos móviles de forma generalizada relaciona el significado de brecha digital con la desigualdad en el acceso a internet y el analfabetismo digital. La brecha digital va más allá del acceso a las TIC, se vincula a la existencia de infraestructura de redes, accesibilidad a las aplicaciones digitales y la formación en competencias digitales para hacer uso de la tecnología.

Los problemas de conectividad y la brecha digital que genera es uno de los mayores riesgos que ha detectado el profesorado, en el Gráfico 4 los resultados advierten en uno de los mayores riesgos en la docencia no presencial:

- Brecha digital. La cuestión de cómo adaptar la educación obligatoria presencial a un modelo online se encuentra en el camino con la brecha digital, en el alumnado y en el profesorado. El $88 \%$ del profesorado ha detectado e identificado la brecha digital como el mayor problema para la conectividad y conseguir el vínculo entre el profesor y el alumno, barrea que impide gestionar los nuevos roles del liderazgo del profesorado y necesarios en la comunicación

Revista de Comunicación y Salud, 2020, Vol. 10, nº 2, pp. 323-342 
Riegos de la comunicación digital en la transición a la docencia no presencial

digital para luchar contra la dispersión, soledad, la desmotivación y la sensación de esfuerzo excesivo del alumnado.

- Aprendizaje a distancia. Confiar en el alumnado en la gestión de su tiempo de forma autodidáctica, comprometido con las tareas y progresando en solitario, esta es la percepción del $12 \%$ del profesorado.

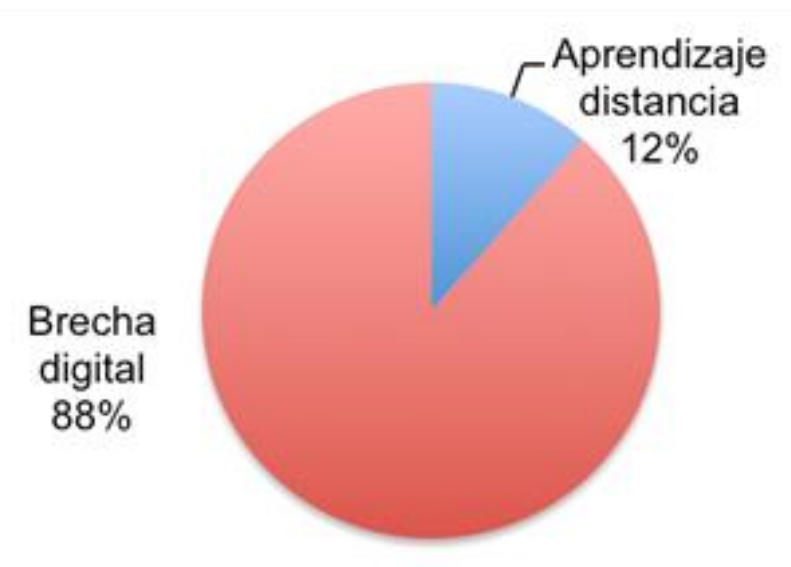

Grafico 4: Conectividad y brecha digital

Fuente: Elaboración propia

Las nuevas tecnologías de la comunicación definitivamente se quedan en la educación, la gran revolución es la nueva conciencia de aprender a aprender, para abrir nuevos canales de comunicación. El profesorado percibe un futuro próximo donde los IES dispondrán de los medios adecuados para la disminución de la brecha digital.

\section{CONCLUSIONES}

El objetivo general de la investigación, analizar el proceso de adaptación de la modalidad de docencia presencial a la modalidad de docencia no presencial, de la actividad docente, en los centros educativos de Formación Profesional durante el confinamiento por el estado de emergencia sanitaria producida por el COVID-19, ha permitido acercarse a la realidad educativa durante la pandemia, donde profesorado y alumnado han cambiado los espacios del aula de los IES para crear nuevos espacios desde sus hogares, estableciendo un feedback a través de las TIC.

Por medio de un cuestionario online el profesorado ha expresado sus percepciones sobre el desarrollo de la docencia y el aprendizaje del alumnado en la modalidad no presencial, información que ha sido analizada, siguiendo los objetivos específicos de la investigación, para averiguar los riesgos de la comunicación digital:

- La docencia no presencial conlleva la transición del rol de transmisor de contenidos al de crear espacios virtuales de comunicación. Para el $84 \%$ del profesorado, el docente como transmisor de información ha dado paso al profesor que fundamenta su labor en la comunicación digital para luchar contra la desmotivación, dispersión y el abandono del aprendizaje por parte del alumnado,

Revista de Comunicación y Salud, 2020, Vol. 10, nº 2, pp. 323-342 
Riegos de la comunicación digital en la transición a la docencia no presencial

e iniciar una gestión del aula virtual basada en la monitorización de las nuevas aplicaciones digitales.

- Los problemas de conectividad genera uno de los riesgos mayores en el nuevo escenario del proceso de enseñanza-aprendizaje, la brecha digital. El el $88 \%$ del profesorado relaciona brecha digital con la inexistencia de infraestructuras de redes, carencia para disponer de dispositivos con accesibilidad a las aplicaciones digitales y la formación de competencias digitales. La brecha digital pone en riesgo la eficiencia de la comunicación digital para el profesorado y para el alumnado.

- El $60 \%$ de los profesores confía en la autorregulación del aprendizaje del alumnado, demostrando la gestión del cambio a través de la adaptación al nuevo escenario digital, esforzándose en mantener el vínculo. Sin embargo esta tendencia no se puede generalizar a todos los grados de FP debido a la heterogeneidad del alumnado de FP Básica, Grado Medio y Grado Superior.

- La mayoría del profesorado, el 56\%, ha mantenido el vínculo con el alumnado por medio del correo electrónico, iniciando una relación de docencia a distancia, el $32 \%$ ha conseguido utilizar Moodle, plataforma que permite iniciar una comunicación digital y gestión del aprendizaje.

El análisis de los datos obtenidos a través de la codificación, tabulación e interpretación de los cuestionarios, permiten verificar a hipótesis "El grado de comunicación digital influye en el grado de desempeño de la docencia en la modalidad presencial", minimizando los riesgos que amenazan la eficiencia de la docencia online: la brecha digital, el docente como transmisor de contenidos, la desmotivación y abandono del alumnado y el analfabetismo digital.

Parafraseando al profesorado encuestado, el Covid-19 ha conseguido que las TIC se quedan en la educación, la gran revolución consiste es la nueva conciencia de aprender a aprender para crear una didáctica innovadora que forme equipos, con estructuras de comunicación que optimicen la labor del docente como líder del escenario digital.

\section{REFERENCIAS}

Bagán, P; García, M y Moreno, A. (2020). El coronavirus y los efectos sobre la desigualdad en el ámbito educativo. En Grupo de investigación Corona social (Ed.), COVID-19 Caos 2.0: Ensayos desconfinados. Ideas de debate para la post pandemia. (pp. 173-193). España: AnthropiQa 2.0.

Barroso, J. y Cabero, J. (Coords.) (2013). Nuevos escenarios digitales. Las tecnologías de la información y la comunicación aplicadas a la formación y desarrollo curricular. Madrid: Pirámide.

Berganza, M.R. y Ruiz, J.A. (2005). Investigar en comunicación. Guía práctica de métodos y técnicas de investigación social en comunicación. Madrid: McGraw-HILL 
Riegos de la comunicación digital en la transición a la docencia no presencial

BlinkLearning (2020). V Estudio sobre el uso de las TIC en la Educación. Disponible en: https://www.blinklearning.com/home?blinklang=MX

Cabrera, E. (2020). La crisis como motor del cambio social. Aspectos educativos fortalecidos tras el confinamiento por la pandemia. En Vázquez, A. y Cambero, S., (Ed.), Reflexiones desconfinadas para la era posCOVID-19. (pp. 193-208), AnthropiQa 2.0.

European Commission. (2006). The European Framework for the Digital Competence of Educators (DigCompEdu). Disponible en: https://ec.europa.eu/irc/digcompedu

Fundación Telefónica. (2016). La sociedad de la Información en España 2016. Madrid: Ariel.

Gobierno de España. Ministerio de la Presidencia, Relaciones con las Cortes y Memoria Democrática. Ley Orgánica 4/1981, de 1 de junio, de los estados de alarma. Boletín oficial del estado no 134 . Disponible en: https://www.boe.es/eli/es/lo/1981/06/01/4/con

Gobierno de España. Ley Orgánica 5/2002 de 19 de junio, de las Cualificaciones y de la Formación Profesional. Boletín oficial del estado, 147, de 20/06/2002. Disponible en: https://www.boe.es/buscar/act.php?id=BOE-A-2002-12018

Gobierno de España. Ley Orgánica 2/2006, de 3 de mayo, de Educación. Boletín Oficial del Estado no 106, 4 de mayo 2006. Disponible en: https://www.boe.es/buscar/act.php?id=BOE-A-2006-7899\&tn=3

Gobierno de España. Ley Orgánica 8/2013, de 9 de diciembre, para la mejora de la calidad educativa. Boletín Oficial del Estado no 295, de 10/12/2013. Disponible en: https://www.boe.es/eli/es/lo/2013/12/09/8/con

Gobierno de España. Ministerio de la Presidencia, Relaciones con las Cortes y Memoria Democrática. Real Decreto 463/2020, de 14 de marzo, por el que se declara el estado de alarma para la gestión de la situación de crisis sanitaria ocasionada por el COVID-19. Boletín oficial del estado № 67. (pp. 25390- 25400). Disponible en: https://www.boe.es/eli/es/rd/2020/03/14/463

Gobierno de España. Ministerio de Educación y Formación Profesional. Orden EFP/365/2020, de 22 de abril, por la que se establecen el marco y las directrices de actuación para el tercer trimestre del curso 2019-2020 y el inicio del curso 20202021, ante la situación de crisis ocasionada por el COVID-19. . Boletín oficial del estado no 114. Disponible en: https://www.boe.es/eli/es/0/2020/04/22/efp365/con

Gobierno de España. Ministerio de Educación y Formación Profesional Orden EFP/361/2020, de 21 de abril, por la que se adoptan medidas excepcionales en materia de flexibilización de las enseñanzas de Formación Profesional del Sistema Educativo y de las enseñanzas de Régimen Especial. Boletín oficial del estado no 113. (pp. 29617 a 29621). Disponible en: https://www.boe.es/eli/es/o/2020/04/21/efp361

Revista de Comunicación y Salud, 2020, Vol. 10, oㅡ 2, pp. 323-342 
Riegos de la comunicación digital en la transición a la docencia no presencial

Gómez, M., Contreras, L., y Gutiérrez, D. (2016). El impacto de las tecnologías de la información y la comunicación en estudiantes de ciencias sociales: un estudio comparativo de dos universidades públicas. Innovación educativa (México, DF), 16(71), 61-80. Disponible en: http://www.scielo.org.mx/scielo.php?script=sci arttext\&pid=S166526732016000200061\&lng=es\&tlng=es

Lankshear, C. y Knobel, M. (2009). Nuevos alfabetismos. Su práctica cotidiana y el aprendizaje en el aula. Barcelona: Ediciones Morata, S.L.

López, F. (2020). El currículo y la educación en el siglo XXI. La preparación del futuro y el enfoque por competencias. Madrid: Narcea.

Pérez, A. (2017). Alfabetización mediática, TIC y competencias digitales. Barcelona: UOC (Universitat Oberta de Cataluña)

Romero, L.M. y Rivera, D. (Coords) (2019). La comunicación en el escenario digital. Actualidad, retos y prospectivas. Perú: Pearson.

Sierra, E: (2020). ¿Cómo será el mundo después del coronavirus?. En Vázquez Atochero, A. y Cambero Rivero, S., (Ed.), Reflexiones desconfinadas para la era posCOVID-19. (pp. 247-266). España: AnthropiQa 2.0.

Torrecillas, T., Vázquez, T., Suárez, R. y Fernández L. M. (2020). El papel de los padres en el comportamiento online de menores hiperconectados. Revista Latina de Comunicación Social, 75, 121-148. doi: 10.4185/RLCS-2020-1419

Unesco. (2013). Enfoques estratégicos sobre las TICS en Educación en América Latina y el Caribe. Oficina Regional de Educación para América Latina y el Caribe. Disponible en: http://www.unesco.org/new/fileadmin/MULTIMEDIA/ FIELD/

Universidad de Zaragoza (2020). Guía de la Universidad de Zaragoza para la adaptación a la docencia no presencial y evaluación online. Disponible en: http://www.unizar.es/actualidad/vernoticia ng.php?id=53801\&idh

Universidad de Zaragoza (2020). Practicum II, especialidad Administración, Marketing, turismo, Servicios a la Comunidad y Formación y Orientación laboral. Disponible en: https://estudios.unizar.es/estudio/asignatura?anyoacademico $=2020$ \&asignatura id=6

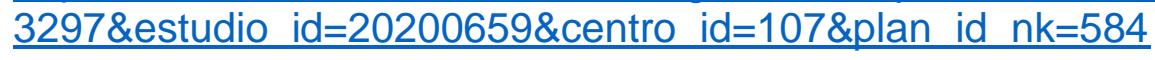

Wood, P. y Smith, J. (2018). Investigar en educación, conceptos básicos y metodología para desarrollar proyectos de investigación. Madrid: Narcea. 
Riegos de la comunicación digital en la transición a la docencia no presencial

\begin{abstract}
AUTOR
Jesús Cuevas Salvador

Licenciatura en Ciencias Políticas y Sociología y Doctorado por la Universidad de Zaragoza. Actualmente es profesor en el Máster en Profesorado en la Facultad de Educación en la Universidad de Zaragoza. Ha realizado estancias de investigación en la Facultad de Ciencias Políticas y Sociología de la Universidad Complutense de Madrid. La actividad investigadora es enfocada en tres líneas de investigación: El Desarrollo Sostenible y la Responsabilidad Social Corporativa de las Organizaciones, la innovación en los procesos de enseñanza-aprendizaje y el análisis de la comunicación digital en la didáctica.
\end{abstract}

Orcid ID: https://orcid.org/0000-0002-6517-8323

Google Scholar: https://scholar.google.es/citations?user=BN78BeoAAAAJ\&hl=es 